Effective silver-assisted welding of YBCO blocks: mechanical versus electrical properties

This article has been downloaded from IOPscience. Please scroll down to see the full text article.

2010 Supercond. Sci. Technol. 23045013

(http://iopscience.iop.org/0953-2048/23/4/045013)

View the table of contents for this issue, or go to the journal homepage for more

Download details:

IP Address: 147.83.132.102

The article was downloaded on 25/01/2013 at 09:13

Please note that terms and conditions apply. 


\title{
Effective silver-assisted welding of YBCO blocks: mechanical versus electrical properties
}

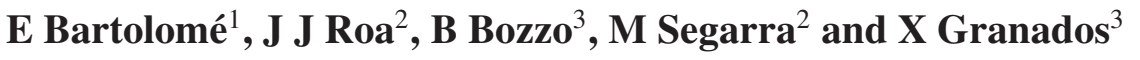 \\ ${ }^{1}$ Escola Universitaria Salesiana de Sarrià (associated with the University Autònoma of \\ Barcelona), Passeig Sant Joan Bosco 74, E-08017 Barcelona, Spain \\ ${ }^{2}$ Centro DIOPMA, Departamento Ciencia de los Materiales e Ingeniería Metalúrgica; \\ Instituto de Nanociencia y Nanotecnología de la Universidad de Barcelona (IN2UB), Facultad \\ de Química, Universidad de Barcelona, Martí i Franqués 1, E-08028 Barcelona, Spain \\ ${ }^{3}$ Institut de Ciència de Materials de Barcelona-CSIC, Campus UAB, E-08193 Bellaterra, \\ Spain
}

Received 2 November 2009, in final form 23 December 2009

Published 10 March 2010

Online at stacks.iop.org/SUST/23/045013

\begin{abstract}
Superconducting welding of bulk YBCO is a key technology allowing the fabrication of large, complex-shaped pieces for applications such as levitation, bearings or large magnets. Ideally, the electrical and mechanical properties of welds should be comparable to that of the joint grains. In this paper, we have investigated the correlation between the microstructural, mechanical and critical current density performances of melt-textured [001]-tilt YBCO welds fabricated by the silver welding technique. The hardness reduction across the weld, measured by nanoindentation, correlates linearly with the decrease of intergranular critical current density, measured at $77 \mathrm{~K}$ and self-field by magnetic Hall mapping. Remarkably, we show that high quality zero-angle welds could be fabricated with unaltered current and hardness performances across the joint, paving the way for the implementation of silver welds in large-scale systems.
\end{abstract}

(Some figures in this article are in colour only in the electronic version)

\section{Introduction}

The performance of some bulk superconducting applications, such as levitation, large magnets or bearings, could be drastically enhanced by using larger $\mathrm{YBa}_{2} \mathrm{Cu}_{3} \mathrm{O}_{7-x}$ (YBCO) blocks. Unfortunately, YBCO pellets of only a few centimetres can be achieved by melt-texturing growth. This fact has motivated the development of alternative procedures to obtain larger tiles, like multi-seeding [1,2] and welding [3] techniques. Silver-assisted welding appears to be the most attractive method in order to build up or repair complex-shaped pieces by assembling primary domains previously fabricated by the standard melting technique; meanwhile, multi-seeding allows us to obtain large pieces during the same growth process. In any case, ideal efficiency is achieved only if the joint pieces are electrically and mechanically comparable to single-domain blocks.

In the scope of this research, we developed a robust superconducting welding technique based on the use of a metallic Ag foil as the welding agent [4]. The process is based on the controlled melting of the foil and diffusion of the $\mathrm{Ag}$ into the $\mathrm{YBCO}$ matrix at an annealing temperature $T_{\max }=985^{\circ} \mathrm{C}$ above the peritectic temperature of the YBCO/Ag composites $\left(T_{\mathrm{p}} \approx 980^{\circ} \mathrm{C}\right)$, but below that of $\mathrm{YBCO}$ $\left(T_{\mathrm{p}} \approx 1010^{\circ} \mathrm{C}\right)$. The use of a silver foil as welding agent presents several advantages: (i) the large temperature window existing among the peritectic temperatures of pure YBCO and $\mathrm{YBCO} / \mathrm{Ag}$ composite facilitates the seeding process of the molten interface from the lateral tiles to be welded; (ii) no dissimilar interfaces are created [5] and (iii) the process is easily scalable.

The silver welding technique enables us to produce both symmetric and asymmetric, [001]-tilt [6] or [110]-tilt [7] superconducting welds in a large range of misorientation angles, as required for flexible fabrication of different complex-shaped parts. Details of the microstructure were reported elsewhere [7]. The magnetic properties of melttextured [001]-tilt YBCO welds have been thoroughly studied 

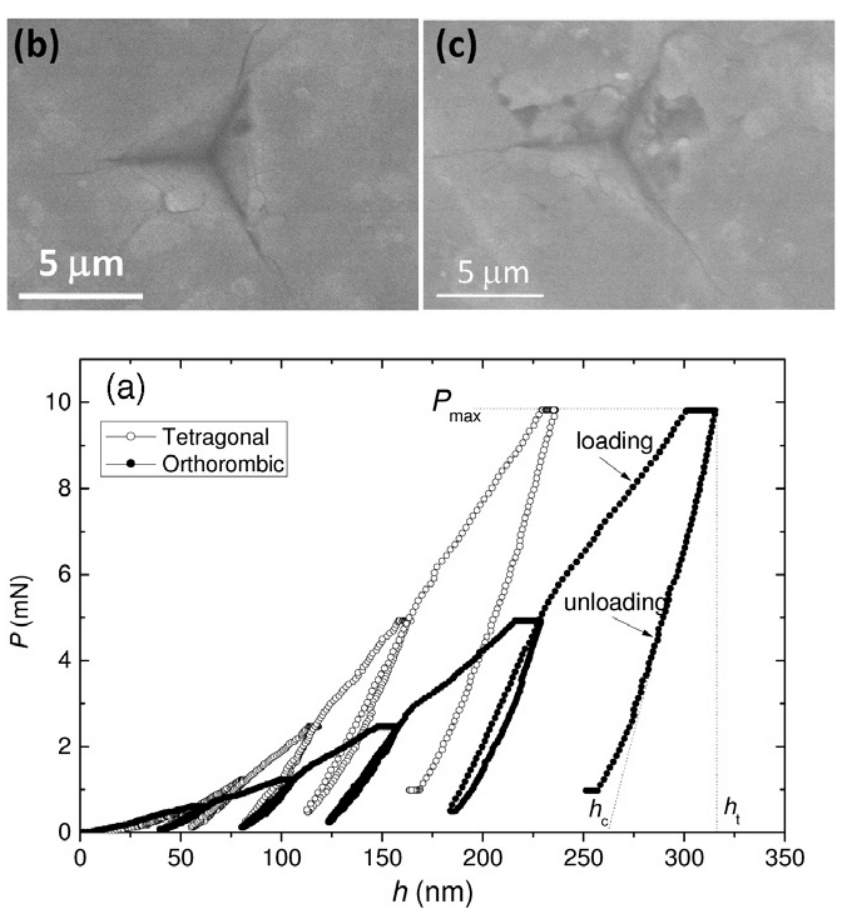

Figure 1. (a) Load versus indenter displacement curves after five loading/unloading processes with a maximum applied force of $P_{\max }=10 \mathrm{mN}$ for reference bulk sample in the tetragonal and orthorhombic phases. On top: the projected area $A_{\mathrm{c}}$ is obtained from an FE-SEM image of the nanoindentation imprint: (b) tetragonal phase and (c) orthorhombic phase.

in the past, using Hall magnetic mapping [8]. The critical current density across the weld, $J_{\mathrm{c}}^{\mathrm{GB}}$, and at the adjacent grains, $J_{\mathrm{c}}^{\mathrm{G}}$, were simultaneously determined as a function of the applied magnetic field at $77 \mathrm{~K}$. Inductive results demonstrated that high quality zero-angle welds could be fabricated, with inter-to-intragrain critical current density ratios $J_{\mathrm{c}}^{\mathrm{GB}} / J_{\mathrm{c}}^{\mathrm{G}} \approx 1$. For misoriented welds, the dependence of the intergrain critical current density on the applied magnetic field and the angle, $J_{\mathrm{c}}^{\mathrm{GB}}(\theta, H)$, was established. Guidelines for the choice of the most adequate $\theta$ weld for each application were provided, as a function of operational field requirements.

It is worth remarking that, for superconducting welds to become functional in applications, mechanical stability in addition to high electrical performance must be guaranteed. Despite that, the mechanical properties of superconducting joints have never been studied.

In this paper, we report results on the mechanical properties of melt-textured YBCO silver welds in correlation with their microstructure and critical current density performance. We determined the nanohardness and Young's modulus across welds of different [001]-tilt angles using a nanoindentation technique at room temperature [9]. In the last decade nanoindentation has become one of the most versatile methods for the characterization of the mechanical properties at the micronanoscale of a broad spectrum of materials [10, 11], among which are YBCO bulk ceramics [12-14] and thin films [15]. Contact phenomena activated during the indentation process at different areas of the weld have been studied by observation of the indentation imprints with field emission scanning electron
Table 1. Experimental parameters for nanoindentations.

\begin{tabular}{ll}
\hline Name & Value \\
\hline Allowable drift rat & $0.05 \mathrm{~nm} \mathrm{~s}^{-1}$ \\
Maximum load & $10 \mathrm{mN}$ \\
Number of loads & 5 \\
Peak hold time & $30 \mathrm{~s}$ \\
Unload percentage & $90 \%$ \\
Time to load & $15 \mathrm{~s}$ \\
\hline
\end{tabular}

microscopy (FE-SEM). The critical current densities across the joints have been determined from quantitative Hall mapping in the remanence at $77 \mathrm{~K}$. We will show that the silver welding technique can provide high quality zero-angle YBCO joints with unaltered current and mechanical properties across the boundary.

\section{Experimental procedures}

YBCO welds with asymmetric [001]-tilt boundaries were prepared by the silver welding technique [3, 4]. Samples were fabricated from commercial YBCO bulk single domains with nominal composition $\mathrm{Y} 123+25 \mathrm{~mol} \% \mathrm{Y}_{2} \mathrm{O}_{3}+$ $1 \mathrm{wt} \% \mathrm{CeO}_{2}$ [16]. $1 \mathrm{~cm}^{3}$ cubes were extracted from the as-grown tetragonal samples. We halved each YBCO cube parallel to the $c$ axis and then made a second cut with a relative misorientation $\theta$ in one of the adjacent grains. The two joining surfaces so obtained were polished with diamond powder down to a size of $1 \mu \mathrm{m}$, and a $10 \mu \mathrm{m}$ thick $\mathrm{Ag}$ foil was introduced between them. The sandwich-like assembling so obtained was fixed between two alumina plates parallel to the joint and placed in a horizontal position in the furnace. The specimen was first heated up to $T_{\max }=995^{\circ} \mathrm{C}$ and kept there for $3 \mathrm{~h}$; then it followed a slow-cooling ramp at $0.6^{\circ} \mathrm{h}^{-1}$ down to $950^{\circ} \mathrm{C}$ and a fast cooling to room temperature. Finally the welded samples were annealed in flowing oxygen at 1.1 bar for $120 \mathrm{~h}$ at $450{ }^{\circ} \mathrm{C}$ to achieve the orthorhombic, superconducting phase. (Details on the optimization of the thermal process can be found in [5].) Samples were halved parallel to the $a b$ plane for measurements, so the final dimensions were $1 \times 1 \times 0.5 \mathrm{~cm}^{3}$. For this study we considered two $\theta=0^{\circ}$ welds (a standard $0^{\circ}$ weld and a non-optimized, low quality one, chosen to test the sensitivity of the technique to variations of $H$ across the weld); two different $\theta=14^{\circ}$ welds (A and B), and a bulk reference sample (without weld) for comparison.

Nanoindentation measurements at room temperature were performed with a Nanoindenter $\mathrm{XP}^{\circledR}$ system (Agilent Technologies) equipped with Test Works 4 Professional level software and a Berkovich tip. In a typical indentation process, the diamond indenter was repeatedly pushed and withdrawn from the material at a given position, and the load $(P)$ versus tip penetration $(h)$ was recorded (figure 1 ). The maximum applied load was chosen as $10 \mathrm{mN}$, small enough to produce net imprints which allowed the accurate measurement of the imprint area $A_{\mathrm{c}}$. At this load, obtained values of $H$ and $E$ correspond to the YBCO compound and not to each of the separate Y123 and Y211 phases [13]. Table 1 summarizes other measurement parameters chosen 
for nanoindentations. The loading/unloading $P-h$ curve characterizes the elastic/plastic response of a sample. The elastic recovery is calculated from the difference between the total indentation depth $\left(h_{\mathrm{t}}\right)$ at maximum indented load $\left(P_{\max }\right)$ and depth of residual impression upon unloading $\left(h_{\mathrm{t}}\right)$.

From the analysis of the $P-h$ curves, the nanohardness $(H)$ and Young's modulus $(E)$ at each point was obtained using the Oliver and Pharr [17] relations:

$$
\begin{gathered}
h_{c}=h_{\mathrm{t}}-\varepsilon \frac{P_{\mathrm{max}}}{S}, \\
A_{\mathrm{c}}=A\left(h_{c}\right)=24.56 \cdot h_{c}^{2}, \\
H=\frac{P}{A_{\mathrm{c}}}, \\
E_{\mathrm{eff}}=\frac{\sqrt{\pi}}{2} \frac{S}{\sqrt{A_{\mathrm{c}}}}, \\
\frac{1}{E_{\mathrm{eff}}}=\frac{1-v_{i}^{2}}{E_{i}}+\frac{1-v^{2}}{E},
\end{gathered}
$$

where $\varepsilon$ is the strain ( 0.75 for the Berkovich indenter), $A_{\mathrm{c}}$ is the projected area under the load, $E_{\text {eff }}$ is the effective Young's modulus, $S$ is the elastic constant stiffness (obtained from the load/unload curve $\mathrm{d} P / \mathrm{d} h), E$ is the Young's elastic modulus, $v$ is Poisson's ratio of the tested YBCO material $(v=0.3[18])$, $E_{i}=1141 \mathrm{GPa}$ and $v_{i}=0.07$ are the elastic modulus and Poisson's ratio of this nanoindenter [9]. The tip was calibrated against a fused silica standard before measurements.

In order to investigate the variation of mechanical properties across the welds, we performed nanoindentation scans on the $a b$ plane in a range of $2 \mathrm{~cm}$ across each boundary. The separation between imprints was $50 \mu \mathrm{m}$ in order to isolate the plastic behaviour generated under the residual imprint (as a general rule, the imprint separation should be 20-30 times the maximum penetration depth). Six hundred indentations were performed for each studied sample in order to achieve statistical significance. Each of the $E$ and $H$ values plotted in subsequent figures is the average of 15 scans performed across the boundary, separated $50 \mu \mathrm{m}$ from each other (figure 1(a)).

Nanoindentation imprints were observed with a field emission Hitachi H-4100 scanning electron microscope (FESEM). These images served a threefold purpose: (i) to discard nanoindentation measurements performed on sample pores; (ii) to measure the contact area $A_{\mathrm{c}}$ required for the $E, H$ determination, thus avoiding over- or underestimations due to sink-in or pile-up events [19] and (iii) to investigate the different fracture mechanisms activated during the indentation process.

A magnetic Hall probe scanning system with $160 \mu \mathrm{m}$ spatial resolution was used to determine $B_{z}(x, y)$ maps of the $a b$ plane of welds in the remanence at $77 \mathrm{~K}$. Details of the system can be found elsewhere [20-22]. The grain and intergrain critical current densities, $J_{\mathrm{c}}^{\mathrm{G}}$ and $J_{\mathrm{c}}^{\mathrm{GB}}$, were simultaneously determined from inversion of the measured $B_{z}$ map, following the methodology described in [23, 24].
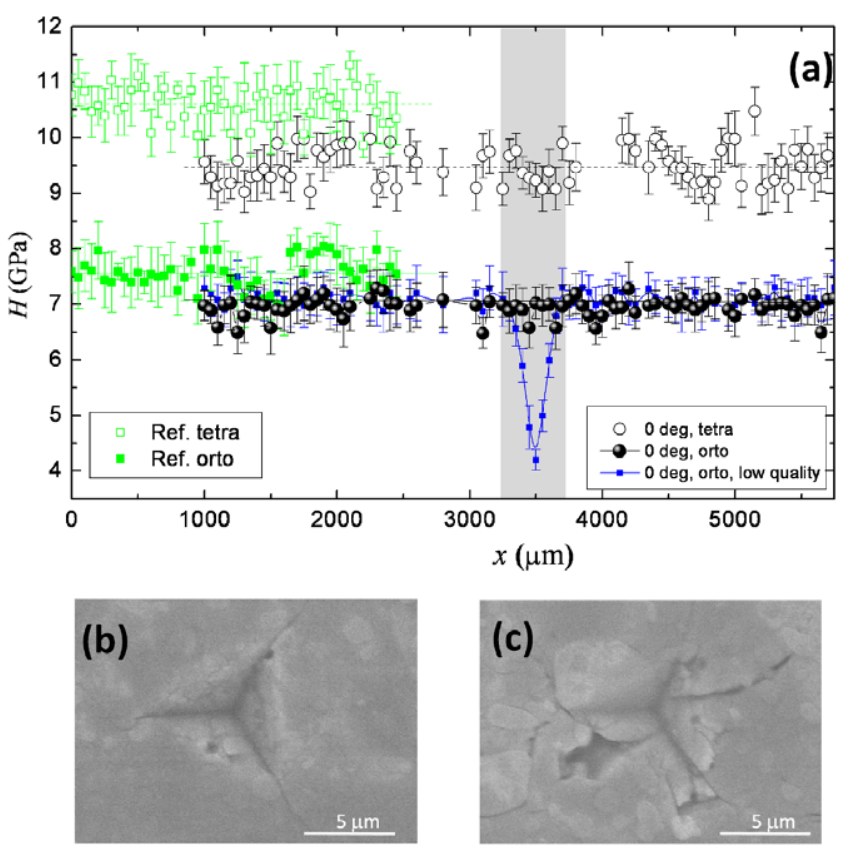

Figure 2. Top: (a) nanohardness scans, $H(x)$, across a high quality $\theta=0^{\circ}$ weld in the tetragonal $(O)$ and orthorhombic $(\bullet)$ phases; $H(x)$ across a low quality $\theta=0^{\circ}$ weld in the orthorhombic phase (๘). The shadowed region indicates the approximate weld position. For comparison, the nanohardness of the bulk reference sample in tetragonal $(\square)$ and orthorhombic ( $\square$ ) phases are shown; below: FE-SEM images of indentation imprints in the weld region for the high quality $0^{\circ}$ weld (b), and the low quality $0^{\circ}$ weld (c).

\section{Results and discussion}

First, the mechanical properties of a melt-textured sample were measured to have a reference of the material characteristics prior to the welding procedure. Figure 1 displays typical loading/unloading $P-h$ curves measured for the reference sample in the tetragonal and orthorhombic phases. As a result of the oxygenation process, the penetration depth in the orthorhombic phase is larger than in the tetragonal phase, and thus, according to equation (3), the hardness is smaller in the former phase. The FE-SEM image of the nanoimprint in the orthorhombic phase (figure 1, inset) shows superficial defects such as cracks at the corners of the imprint and a chipping effect, which were activated during the indentation process.

The nanohardness values for the bulk reference sample in the tetragonal phase, $H=10.6 \pm 0.4 \mathrm{GPa}$, and orthorhombic phase, $H=7.6 \pm 0.4 \mathrm{GPa}$ are shown in figure 2. As expected, the hardness is smaller in the less compact crystalline phase. However, the Young's modulus remains constant in both phases, within the error $(E=120 \pm 5 \mathrm{GPa})$. This value is in good agreement with different values reported in the literature for melt-textured YBCO $[25,26]$ and smaller than those reported for Bridgman-grown YBCO at similar applied loads [13]. The Young's modulus depends basically on the composition of the material, thus $E$ does not change significantly with the crystalline phase, but it is influenced by the texturing process affecting the microstructure.

Figure 2 depicts as well the $H$ measured across a $\theta=0^{\circ}$ weld. We observe a decrease of around $10 \%$ in the grain 

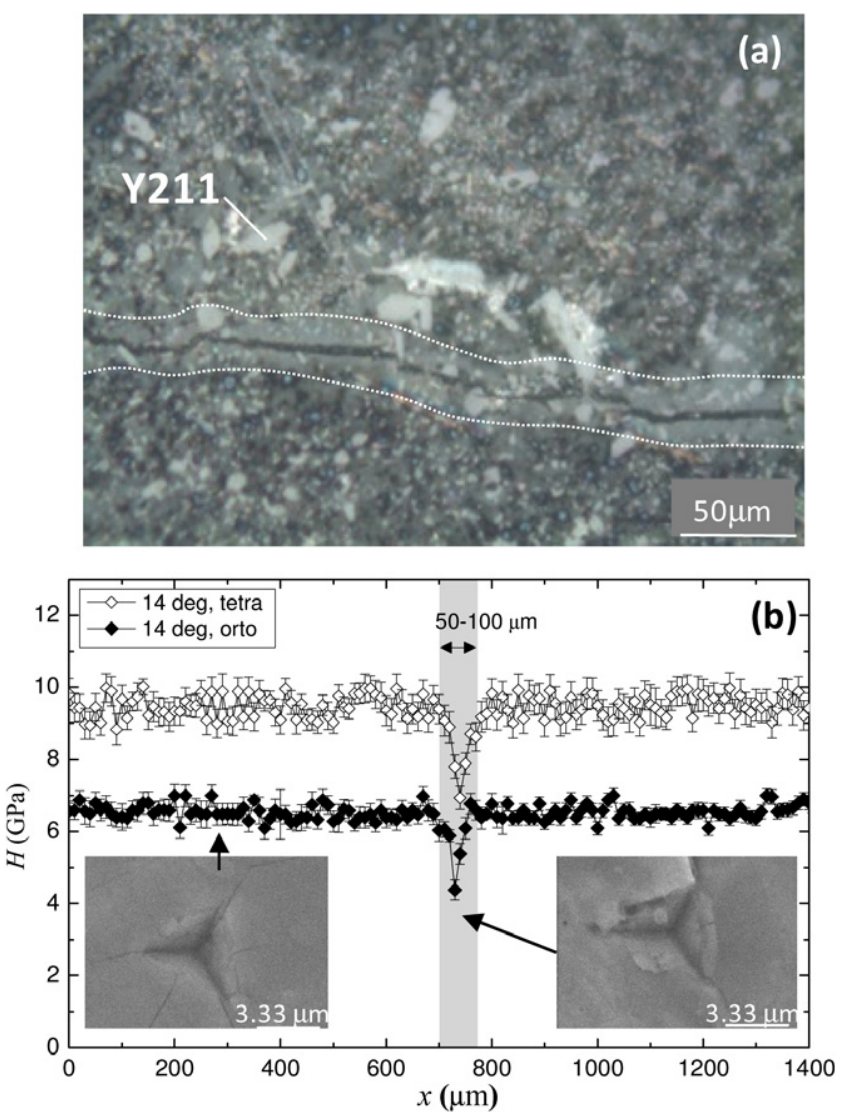

Figure 3. YBCO silver weld with a $\theta=14^{\circ}$ angle. (a) Optical micrograph showing the decrease in the density of Y211 particles in a 'welding path' of around $50 \mu \mathrm{m}$, after oxygenation process; (b) nanohardness scan $H(x)$ across the weld in the tetragonal $(\diamond)$ and orthorhombic phase $(\checkmark)$, and FE-SEM images far from the weld and at the weld region.

nanohardness with respect to the reference sample in the two phases. This fact may be explained by the presence of silver in the YBCO matrix, migrated from the weld region during the thermal treatment. The maximum silver content could be estimated taking into account the welding path width and the amount of silver used for welding, although it was not directly detected in the weld. This percentage has been established in $0.5 \mathrm{wt} \%$ in the $5 \mathrm{~mm}$ scanned region, considering densities of $\mathrm{Ag}$ and $\mathrm{YBCO}$ to be $\rho_{\mathrm{Ag}}=10.4 \mathrm{~g} \mathrm{~cm}^{-3}$ and $\rho_{\mathrm{YBCO}}=$ $4.7 \mathrm{~g} \mathrm{~cm}^{-3}$. It could be possible that the addition of a small percentage of silver decreases the nanohardness for the small contact depths used in our indentations. It has been earlier reported that a $5 \mathrm{wt} \% \mathrm{Ag}_{2} \mathrm{O}$ in $\mathrm{YBCO}$ decreases $H$ for contact depths $h<300 \mathrm{~nm}$, whereas for larger contact depths $H$ was observed to increase [27]. This means that if silver is in the origin of the grain softening, it occurs only in the sample surface.

Remarkably, the nanohardness was not degraded at all across the $0^{\circ}$-weld boundary. In order to verify that our technique was sensitive to variations of $H$ across the weld, we also measured a low quality $0^{\circ}$ weld, obtained after a non-optimized thermal treatment. The degradation of the nanohardness at the weld region can be clearly appreciated in this case. For the high quality $0^{\circ}$ weld, the FE-SEM image of

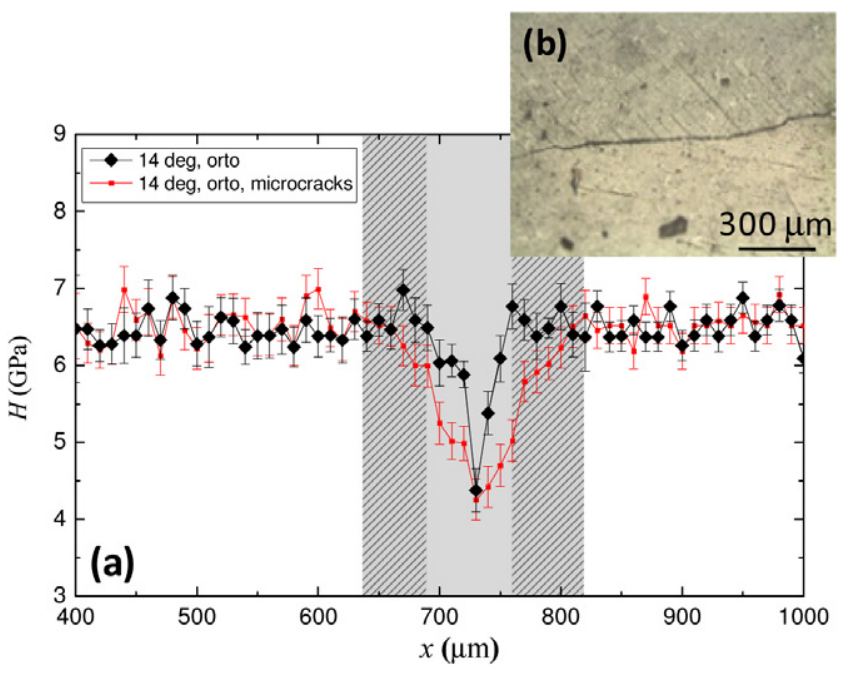

Figure 4. (a) Nanohardness scan $H(x)$ across a $\theta=14^{\circ}$ weld in the orthorhombic phase, in a region (b) affected by microcracks ( $\mathbf{})$. For comparison, $H(x)$ across a region free of microcracks $(\checkmark)$.

the nanoimprint in the weld region (figure 2(b)) looks similar to that in the adjacent grains. In contrast, the imprint in the weld region of the low quality $0^{\circ}$ weld (figure $2(\mathrm{c})$ ) showed incipient chipping effects.

Figure 3 shows the nanohardness measured across a $\theta=$ $14^{\circ}$ weld in the tetragonal and orthorhombic phases. The $H$ values at the grains were similar for all measured welds, in the order of $H=9.42 \pm 0.39 \mathrm{GPa}$ (in tetragonal phase), with a decrease of $25-30 \%$ in the orthorhombic phase. A decrease of $40 \%$ in the hardness in a 50-100 $\mu \mathrm{m}$ region around the weld is observed. A microstructure analysis (figure 3(a)) reveals that the density of Y211 particles vanishes in a welding path of approximately the same width. The existence of this welding path in silver welds has been explained by the higher $\mathrm{Y}$ content in the melt produced by diffusion and dissolving of solid bulk phases in melted Ag during heating, and by leaking of this melt out of the weld [7]. The nanohardness of the Y211 phase is almost a factor of two larger than that of the Y123 phase $\left(H_{\mathrm{Y} 211} \approx 2 . H_{\mathrm{Y} 123}\right)$, as concluded from nanoindentation measurements at very low loads [13]. Thus, the absence of Y211 particles in the welding path produces a reduction of the YBCO compound hardness measured at the weld region.

Occasionally, the oxygenation process induced microcracks in certain weld areas arising from the boundary and extending over widths of the order of 200-1000 $\mu \mathrm{m}$ (figure 4(b)). In that case, the nanohardness was reduced in a region of that size, broader than the welding path (figure 4(a)). Moreover, in some cases large macrocracks developed at both sides of the weld as a result of the oxygenation, as observed in figure 5(a). The nanohardness across the weld decreased then in a broad area of approx. $3 \mathrm{~mm}$ (figure 5(b)). Notice that the $H(x)$ reduction is asymmetric, the steeper reduction corresponding to the grain affected by the major crack. The FE-SEM imprint image in a region affected by macrocracking presents unusual fracture effects (figure 5): there seems to be a stress field dragging material inside the imprint region, but due to the small force applied, there is not enough energy 

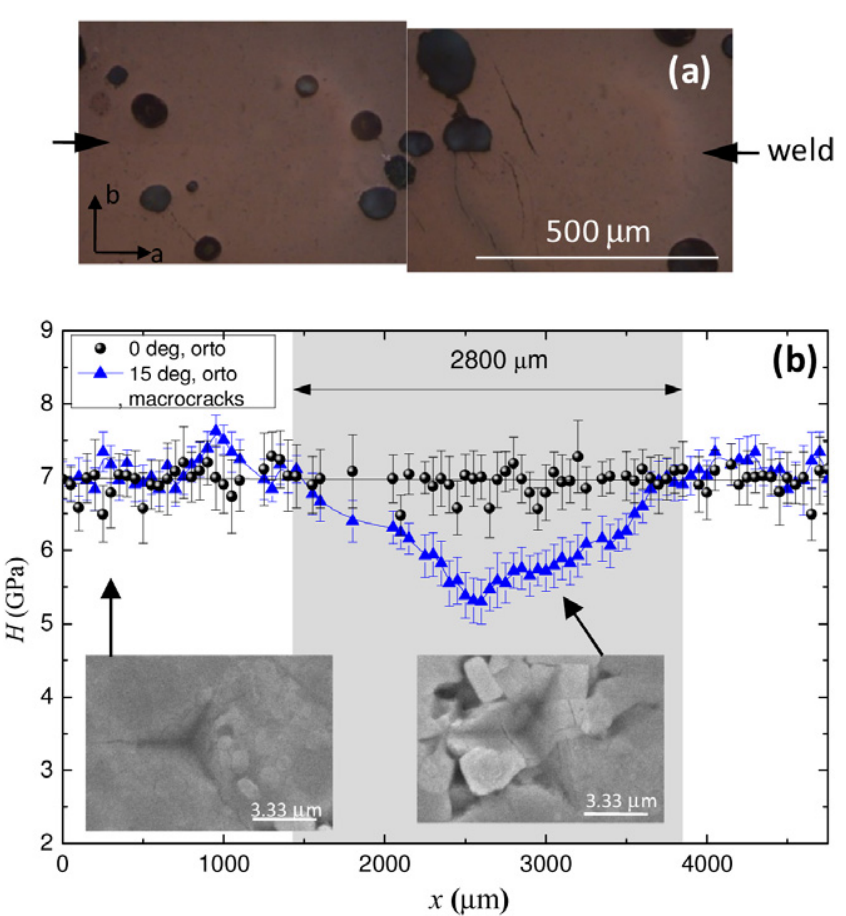

Figure 5. $\theta=14^{\circ}$ (B) weld. (a) Optical micrograph of the $a b$ plane of the oxygenated weld (indicated by arrows), in a region affected by macrocracks; (b) nanohardness scan $H(x)$ across the weld region affected by the microcracks $(\boldsymbol{\Lambda})$. FE-SEM images of the indenter imprints at the grains, and at the grain region.

to produce chipping. This effect is only observed in welds affected by macrocracks and not in neat regions (figure 3 ).

Concerning the Young modulus, values of $E=120 \pm$ $5 \mathrm{GPa}$ (in tetragonal and orthorhombic phases) were measured for all samples, without noticeable variation across the weld. It should be noted that $E$ is basically determined by the interatomic bonds, i.e. the chemical composition of the material, and secondarily, on its inhomogeneous microstructure. The gradient of Y211 particles or presence of micro/macrocracks at the weld region did not significantly modify $E$, within our determination error.

Finally, we studied the relation between the electrical and mechanical performance of YBCO silver welds, the later described in terms of the ratio $H^{\mathrm{GB}} / H^{\mathrm{G}}$ between the minimum nanohardness value at the weld region and that of the grains. The intergrain-to-intragranular critical current density of each weld, $J_{\mathrm{c}}^{\mathrm{GB}} / J_{\mathrm{c}}^{\mathrm{G}}$, was determined from the magnetic field distribution $B_{z}(x, y)$ at $77 \mathrm{~K}$, in the remanence (see, e.g., figure 6), following the procedure already described in [23].

We have found that the nanohardness ratio at the weld $H^{\mathrm{GB}} / H^{\mathrm{G}}$ linearly correlates with the inter-to-intragrain critical current density ratio $J_{\mathrm{c}}^{\mathrm{GB}} / J_{\mathrm{c}}^{\mathrm{G}}$ at $77 \mathrm{~K}$, in self-field (figure 7). The extrapolation of the linear fit tends to the expected nanohardness ratio for a $\mathrm{YBCO} / \mathrm{Ag} / \mathrm{YBCO}$ sandwich sample (with no passage of current across the weld, $J_{\mathrm{c}}^{\mathrm{GB}} / J_{\mathrm{c}}^{\mathrm{G}}=0$ ), of $H^{\mathrm{GB}}(\mathrm{Ag}) / H^{\mathrm{G}}(\mathrm{YBCO}) \approx 1.4 / 7.5=0.2$. This result has a potential practical implication: by measuring a mechanical property at room temperature, one can obtain the current performance in the superconducting state, thus avoiding the

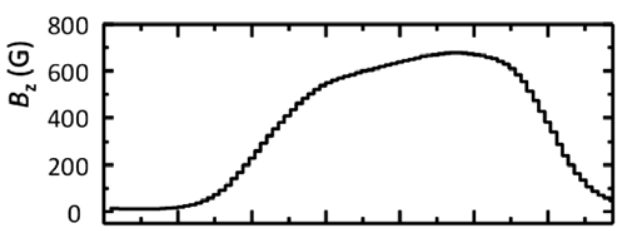

$B_{z}(G)$

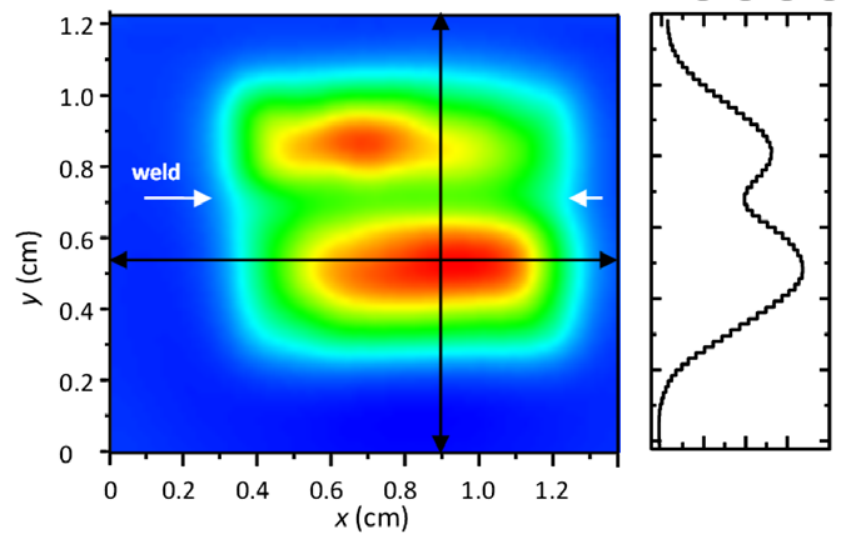

Figure 6. Magnetic field distribution $B_{z}(x, y)$ of a $\theta=14^{\circ}$ (B) weld measured in the remanence at $77 \mathrm{~K}$ with a Hall scanning microscope.

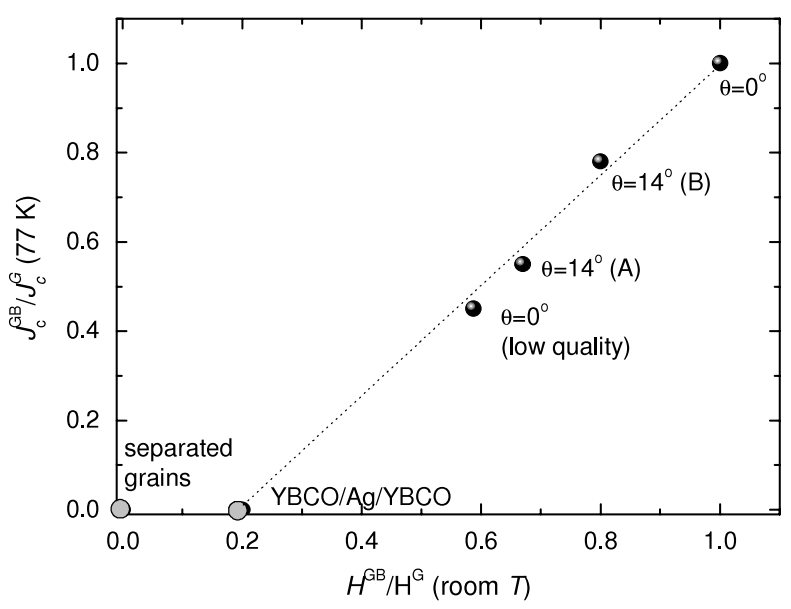

Figure 7. Correlation between the inter-to-intragranular mechanical nanohardness at room temperature $\left(H^{\mathrm{GB}} / H^{\mathrm{G}}\right)$ obtained from nanoindentation scans, and critical current density $\left(J_{\mathrm{c}}^{\mathrm{GB}} / J_{\mathrm{c}}^{\mathrm{G}}\right)$ at $77 \mathrm{~K}$ determined by quantitative magnetic Hall microscopy. The linear fit extrapolates to the nanohardness ratio of a $\mathrm{YBCO}-\mathrm{Ag}-\mathrm{YBCO}$ sandwich sample, $H^{\mathrm{GB}}(\mathrm{Ag}) / H^{\mathrm{G}}(\mathrm{YBCO}) \approx 0.2$.

use of cryogenics tests which are expensive and cumbersome to perform in industry.

For the non-disoriented weld, the critical current density and the mechanical properties across the weld remain unchanged $\left(H^{\mathrm{GB}} / H^{\mathrm{G}}=1, J_{\mathrm{c}}^{\mathrm{GB}} / J_{\mathrm{c}}^{\mathrm{G}}=1\right)$. This result shows that mechanically robust, high quality $0^{\circ}$ welds for system applications can be readily achieved by the silver welding methodology.

In this work we presented nanoindentations at room temperature. This represents an initial, non-destructive way of evaluating the mechanical properties of welds, which already provides useful information about the superconducting current performance across the weld through the correlation 
function shown in figure 7 . In the future, more work should be done to gain knowledge of the mechanical properties of welds at superconducting temperatures. Nanoindentation at cryogenic temperatures still presents many technological problems. However, tensile [28] and three-point bending [29] tests could be used to deduce the Young's modulus, fracture strength and toughness at superconducting temperatures.

\section{Conclusions}

Superconducting joining represents a key enabling technology for the fabrication of large YBCO blocks, allowing an enhancement in the performance of several large-scale applications. Efficient joints must prove not only high electrical connectivity but also mechanical stability. We have evaluated the mechanical and critical current density properties of melt-textured YBCO welds fabricated by silver welding.

The nanoindentation technique has been demonstrated to be a powerful technique to characterize the mechanical properties of superconducting joints at room temperature. We determined the variation of the nanohardness and Young's modulus across the $a b$ plane of YBCO silver welds of different [001] misorientation angles. A reduction of $30 \%$ in the value of the grain nanohardness is measured as the sample is oxygenated and passes from tetragonal to the orthorhombic phase. For misoriented welds the nanohardness is reduced in a $50 \mu \mathrm{m}$ width welding path along the boundary due to the absence of the harder Y211 phase. The oxygenation process induces in some cases micro- and macrocracks arising from the weld, which decrease the nanohardness in a broader area ranging between $100 \mu \mathrm{m}$ and $3 \mathrm{~mm}$, respectively. The decrease in nanohardness across the weld correlates with the intergranular reduction of critical current density, determined from Hall magnetic microscopy at $77 \mathrm{~K}$. Interestingly, high quality zero-angle welds could be fabricated without degradation of either their mechanical or magnetic properties across the joint. This represents an important step forward towards the implementation of welds in bulk, largescale systems.

\section{Acknowledgments}

We acknowledge the financial support from MICINN (MAT2008-01022, NAN2004-09133-CO3-01, Consolider NANOSELECT), Generalitat de Catalunya (Catalan Pla de Recerca 2009-SGR-770 and XaRMAE) and EU (HIPERCHEM and NESPA). We would like to thank W Boch from NEXANS Superconductors for providing the bulk material.

\section{References}

[1] Jee Y A, Kim C J, Sung T H and Hong G W 2000 Supercond. Sci. Technol. 13195

[2] Kim C J et al 2000 Physica C 338205

[3] Iliescu S et al 2004 Supercond. Sci. Technol. 17182

[4] Iliescu S, Granados X, Puig T and Obradors X 2006 J. Mater. Res. 212534

[5] Iliescu S et al 2005 Supercond. Sci. Technol. 18 S168

[6] Bozzo B et al 2006 J. Phys. Conf. Proc. 43401

[7] Sefcikova M et al 2008 Mater. Sci. Eng. B 151107

[8] Bartolome E et al 2008 Supercond. Sci. Technol. 2112502

[9] Pharr G M 1998 Mater. Sci. Eng. A 253151

[10] Loubet J L et al 1984 Trans. ASME, J. Tribol. 10643

[11] Doener M F and Nix W D 1986 J. Mater. Res. 1601

[12] Goyal A et al 1991 Physica C $\mathbf{1 8 3} 221$

[13] Roa J J et al 2007 Nanotechnology 18385701

[14] Lim Y Y et al 2001 J. Phys. D: Appl. Phys. 34 L70

[15] Soifer Ya M et al 2004 Physica C 40280

[16] Ullrich M et al 1999 Physica C 31186

[17] Oliver W and Pharr G 1992 J. Mater. Res. 71564

[18] Xiong J et al 2007 J. Cryst. Growth 300364

[19] Giannakopoulos A E and Suresh S 1990 Scr. Mater. 401191

[20] Granados X et al 2003 IEEE Trans. Appl. Supercond. 133667

[21] Bartolomé E et al 2004 Phys. Rev. B 70144514

[22] Bartolomé E et al 2005 Phys. Rev. B 72024523

[23] Bozzo B et al 2005 Supercond. Sci. Technol. 181227

[24] Granados X et al 2005 IEEE Trans. Appl. Supercond. 153632

[25] Lucas B N et al 1996 J. Mater. Res. 62519

[26] Giese R F et al 1990 Proc. Am. Power Conf. (Chicago, Apr. 1990) 52 776-92

[27] Foerster C E et al 2008 Braz. J. Phys. 38341

[28] Okudera Tet al 2003 Physica C 392628

[29] Sato Tet al 2006 Physica C 445422 Katagiri K et al 2006 Supercond. Sci. Technol. 19 S545 